\title{
Comparing Years of Healthy Life, Measured in 16 Ways, for Normal Weight and Overweight Older Adults
}

\author{
Paula Diehr, ${ }^{1,2}$ Stephen Thielke, ${ }^{3,4}$ Ellen O'Meara, ${ }^{5}$ \\ Annette L. Fitzpatrick, ${ }^{6}$ and Anne Newman ${ }^{7}$ \\ ${ }^{1}$ Department of Biostatistics, University of Washington, Seattle, WA 98195, USA \\ ${ }^{2}$ Department of Health Services, University of Washington, Seattle, WA 98195, USA \\ ${ }^{3}$ Department of Psychiatry, University of Washington, Seattle, WA 98195, USA \\ ${ }^{4}$ Geriatric Research, Education, and Clinical Center, Puget Sound VA Medical Center, Seattle, WA 98195, USA \\ ${ }^{5}$ Group Health Research Institute, Seattle, WA 98195, USA \\ ${ }^{6}$ Department of Epidemiology, University of Washington, Seattle, WA 98195, USA \\ ${ }^{7}$ Department of Epidemiology and Center for Aging and Population Research, University of Pittsburgh, \\ Pittsburgh, PA 15213, USA \\ Correspondence should be addressed to Paula Diehr, pdiehr@uw.edu
}

Received 14 November 2011; Revised 17 February 2012; Accepted 7 March 2012

Academic Editor: Francisco Lopez-Jimenez

Copyright ( 2012 Paula Diehr et al. This is an open access article distributed under the Creative Commons Attribution License, which permits unrestricted use, distribution, and reproduction in any medium, provided the original work is properly cited.

\begin{abstract}
Introduction. The traditional definitions of overweight and obesity are not age specific, even though the relationship of weight to mortality is different for older adults. Effects of adiposity on aspects of health beside mortality have not been well investigated. Methods. We calculated the number of years of healthy life (YHL) in the 10 years after baseline, for 5,747 older adults. YHL was defined in 16 different ways. We compared Normal and Overweight persons, classified either by body mass index (BMI) or by waist circumference (WC). Findings. YHL for Normal and Overweight persons differed significantly in $25 \%$ of the comparisons, of which half favored the Overweight. Measures of physical health favored Normal weight, while measures of mental health and quality of life favored Overweight. Overweight was less favorable when defined by WC than by BMI. Obese persons usually had worse outcomes. Discussion. Overweight older adults averaged as many years of life and years of healthy life as those of Normal weight. There may be no outcome based reason to distinguish Normal from Overweight for older adults. Conclusion. The "Overweight paradox" appears to hold for nonmortality outcomes. New adiposity standards are needed for older adults, possibly different by race and sex.
\end{abstract}

\section{Introduction}

Standard definitions of overweight and obesity, based on body mass index (BMI), do not differ by age [1]. However, many studies of older adults have found a U-shaped relationship between BMI and mortality, with the lowest mortality in the group labeled as "overweight" (BMI from 25 to 29.9) [2]. This surprising finding is often called the "Obesity Paradox." The work in [2] identifies several related research issues, including the two that are addressed here. First, BMI may not measure adiposity well in older adults, and analyses based on waist circumference (WC) may result in less paradoxical results [2]. Second, even if Overweight older adults live as long as persons with Normal BMI, they may spend more of those years being sicker, more disabled, or with worse physical function. This paper will attempt to provide insight into both of those issues.

We conducted a longitudinal study to measure the relation of adiposity to 16 domains of health in older adults, using both BMI and WC to classify adiposity. We hypothesized that Overweight older adults, whether classified by BMI or WC, would have as many years of healthy life (YHL) and years of life (YOL) as those classified as Normal weight. In other words, we expected the Obesity Paradox (perhaps, more aptly, the "Overweight Paradox") to hold for health status as well as for mortality. Obese older adults were expected to have fewer (worse) YHL than persons with Normal WC. We hypothesized that results for WC would be 
similar to those for BMI. We also explored whether results differed for men and women, blacks and whites, and for different measures of health status.

\section{Methods}

\subsection{Data}

2.1.1. Study Sample. Data came from the Cardiovascular Health Study (CHS), a population-based longitudinal study of risk factors for heart disease and stroke in 5888 adults aged 65 and older at baseline [3]. Participants were recruited from a random sample of Medicare eligible persons in four U.S. communities, and extensive data were collected during annual clinic visits and telephone calls. The original cohort of 5201 participants, recruited in 1989-1990, had up to ten annual clinic examinations. A second cohort of 687 African Americans, from 3 of the original study communities, were enrolled in about 1992-1993 and had up to seven annual examinations. Followup is ongoing for mortality.

Exclusions. We excluded 19 persons who were missing baseline BMI, 44 who identified themselves as neither black nor white, and 22 more who were missing one or more key baseline variables. We also removed the 97 persons with $\mathrm{BMI}<18.5$ (Underweight), because of their small numbers. The current study involves the remaining 4830 whites (followed for 10 years) and 904 blacks (the second cohort, plus 217 from the original cohort, all followed for 7 years).

Missing Data. Mortality was verified using CMS records, and is believed to be complete. Followup for other longitudinal variables was also satisfactory [4]. For example, in the current research, in the final study year, 95\% of the subjects either had an observed value for activities of daily living or had died. (Missingness differed somewhat by variable). Missing longitudinal data were imputed by interpolation between two known values where possible. Otherwise the missing value was imputed from the person's last available value, selfrated health, and eventual date of death, as detailed in the appendix.

2.1.2. Measures of Adiposity (Independent Variables). Height, weight, and waist circumference (WC) were measured in the clinic. Persons wore a hospital gown and no shoes, and a calibrated scale was used. Waist circumference (in $\mathrm{cm}$ ) was measured over bare skin over the widest circumference above the iliac crest using a metal tape measure. Body mass index was calculated as weight in kilograms divided by the square of height in meters. Adiposity was first categorized by BMI, using standard thresholds: Normal (18.5-24.9), Overweight (25-29.9), or Obese (30+) [1, 5]. (Persons with BMI below 18.5 had already been excluded). Only 317 (17\%) of the 1854 Obese persons had $\mathrm{BMI}>35$, meaning that most persons classified as Obese had class 1 obesity.

Waist circumference (WC) thresholds of $88 \mathrm{~cm}$ for women and $102 \mathrm{~cm}$ for men have been proposed, but there is no evidence that these are appropriate for older adults
$[1,2,6]$. To categorize WC in a manner comparable to BMI, we chose thresholds to create three groups of equal size (tertiles), referred to for convenience as Normal WC, Overweight WC, and Obese WC. To ensure adequate numbers in each category, tertiles were defined separately for white women, black women, and men. For white women, Normal WC was $<84.5 \mathrm{~cm}$, Overweight WC was 84.5 to 96.4 , and Obese WC was $>96.4 \mathrm{~cm}$. The comparable thresholds were 94.0 and 107.5 for black women and 93.0 and 101.5 for men. About $65 \%$ of persons were in the same adiposity category for both BMI and WC. There were a few major discrepancies: 21 persons had Obese BMI with Normal WC, and 56 had Normal BMI with Obese WC. The two measures of adiposity were thus similar but not identical. The average WC for persons with BMI below 18.5 was about $13 \mathrm{~cm}$ lower than the mean WC for Normals. Thus, the exclusion of the underweight BMI subjects also removed persons with low WC.

2.1.3. Outcome Measures (Dependent Variables). Sixteen definitions of YHL were used in this study as the study outcomes, calculated from the common descriptors of health status listed in Table 1. The variables, measured annually, address the domains of physical function, mental and emotional health, social health, health behaviors and quality of life. Cognition, timed walk, and hospitalization were determined objectively; the others came from patient report. Each value was dichotomized into Healthy (1) or Sick (0), using the thresholds shown in Table 1. If standard thresholds were not available, we chose intuitive thresholds that ensured sufficient data at each level. Persons dead at the time of the measure were coded as 0. For example, to dichotomize ADL, a person with no ADL difficulties was coded as 1 for that observation, while a person with difficulties or who had died was coded as 0 .

The outcome measures for each person, calculated separately for each health variable, were the sum over time of their values, which may be interpreted as the number of years in which the person was healthy (by each definition), during the period starting 6 months before baseline to 6 months after study end. For convenience, we usually refer simply to YHL, without specifying which measure of health being used. The possible range of YHL was 0 to 10 years for whites and 0 to 7 years for blacks. Survival, or years of life (YOL), is a special case of YHL. As an example, a person who was alive at 8 of the 10 measurement times and was healthy with respect to ADL (had no ADL difficulties) at six times (not necessarily consecutive), would have YOL $=8$ and YHL (from ADL) $=6$. YHL does not account specifically for trends; for example, 3 healthy years followed by 3 sick yields the same YHL as 3 sick years followed by 3 healthy.

2.1.4. Covariates. Older age, smoking, and recent weight loss are usually related both to worse health and to lower weight, and are thus potential confounders. All regression analyses were adjusted for baseline age, smoking history, and whether the person had lost 10 or more pounds in the year prior to baseline. Smoking was coded 1 for never smoker, 2 for former smoker, and 3 for current smoker. 
TABLE 1: Definitions of "healthy" based on 16 health-related variables.

\begin{tabular}{|c|c|c|}
\hline Label & Measure & Definition of "Healthy" \\
\hline YOL & Years of life (survival) & Alive \\
\hline SPL & Satisfaction with the Purpose of Life & A score of 1 to 4 on a 10-point scale rating satisfaction with the purpose of life \\
\hline FLW & Feeling about life as awhole [7] & A score of 1 to 3 on a 6 -point scale \\
\hline SOC & Social support $[8]$ & Fewer than 8 points on the 24 -point social support score \\
\hline DEP & Depression & Center for Epidemiologic Studies Short Depression score < $10[9]$ \\
\hline BED & Bed days & No days spent in bed in the previous two weeks \\
\hline FLUSH & Flu shot & Had a flu shot in the previous year \\
\hline COG & Cognition & A modified mini mental state examination score above $89[10]$ \\
\hline HOSP & Hospitalization & Not hospitalized in the previous year \\
\hline EVGG & Self-rated Health & In excellent, very good, or good self-rated health \\
\hline LEV & Stressful life events (10 point modified) & $\begin{array}{l}\text { Event stress score }<2 \text { [11]. Life events, occurring either to the person or } \\
\text { significant other, include moving, death (of significant other), illness, } \\
\text { worsening relationship, financial changes, robbery, caregiving, job change, } \\
\text { new grandchild, or other stressful event. }\end{array}$ \\
\hline EXSTR & Extremity strength & No problems with lifting, reaching, or gripping \\
\hline IADL & Instrumental activities of dailyliving & $\begin{array}{l}\text { No difficulties with instrumental activities of daily living-heavy or light } \\
\text { housework, shopping, meal preparation, money management, or telephoning }\end{array}$ \\
\hline BLOCK & Blocks walked & Walking more than 4 blocks per day, on average [12] \\
\hline $\mathrm{ADL}$ & activities of dailyliving & $\begin{array}{l}\text { No difficulties with activities of daily living-walking, transferring, eating, } \\
\text { dressing, bathing, or toileting }\end{array}$ \\
\hline TWLK & Timed Walk & Able to walk 15 feet in less than 10 seconds (measured) \\
\hline
\end{tabular}

2.2. Analysis. The primary analysis was a regression of each measure of YHL (the dependent variables) on dummy variables representing Overweight and Obese BMI (or WC), controlling for the covariates of baseline age, log age, smoking, and weight loss. (Two age terms were used to permit nonlinear relationships). For example, when YHL is defined as years without ADL difficulties, the regression equation was

$$
\begin{aligned}
\text { YHL }(\text { from ADL })= & b_{0}+b_{1} \text { Overweight }+b_{2} \text { Obese }+b_{3} \text { Age } \\
& +b_{4}(\text { log age })+b_{5} \text { Smoking }+b_{6} \text { Wt Loss. }
\end{aligned}
$$

Overweight and Obese were thus compared to the reference category (Normal). Because we used linear regression, the coefficient for Overweight $\left(b_{1}\right)$ is the adjusted difference in YHL between Overweight and Normal, measured in years. Preliminary analyses found strong and significant interactions between sex, race, and adiposity. For clarity, all regressions were performed separately by sex and race. Separate regressions were performed for each variable, within each sex and race subgroup, using both BMI and WC as the measure of adiposity. There were thus 128 separate regressions comparing Overweight to Normal weight (16 YHL variables $\times 4$ sex/race groups $\times 2$ measures of adiposity). The regressions may be thought of in some sense as replicate analyses. Although YHL is probably not normally distributed (YHL cannot be greater than 10 for the white group or than 7 for the black group), the sample size was large enough for the central limit theorem to guarantee that the regression coefficients would be normally distributed, making linear regression appropriate [13]. The regression coefficients for Overweight were graphed and tabulated. The coefficients for Obese are mentioned only briefly.

\section{Findings}

3.1. Descriptive Statistics. Table 2 shows the means and standard deviations (s.d.) of all the variables, by sex and race. For example, there were 2717 white women, whose mean age was 72.4 (s.d. = 5.4), mean BMI was 26.5, and mean WC was $91.1 \mathrm{~cm}$. Further, 12.1\% were smokers and $11.4 \%$ had lost 10 or more pounds in the previous year. The table lines labeled YOL through TWLK present the mean YHL for each definition of "healthy." For example, the mean number of years the women survived in the 10-year period (YOL) was 9.1, and 7.2 of those years were spent with no ADL difficulties, on average. The 16 measures of YHL have different means, due primarily to differences in how the 16 thresholds for "healthy" were defined (see Table 1). There are also apparent differences among the sex and race subgroups.

Table 3 shows the variable means by BMI category for the largest subgroup, white women. (Table 5 in the appendix has similar information for the other subgroups, and Table 6 in the appendix has the same information categorized by WC instead of BMI). For example, of the 1037 white women with Normal BMI at baseline, the mean age was 73.0 years, $16 \%$ were current smokers, and $13 \%$ had lost 10 or more pounds in the previous year. Normal weight (versus Overweight) was significantly associated with higher age and smoking $(P<$ .001 by Anova, not shown), but weight loss was not ( $P=$ .308). For WC categories (Table 6 in the appendix), Normal 
TABLe 2: Means and standard deviations.

\begin{tabular}{|c|c|c|c|c|c|c|c|c|}
\hline \multirow{3}{*}{ Name } & \multicolumn{4}{|c|}{ White } & \multicolumn{4}{|c|}{ Black } \\
\hline & \multirow{2}{*}{$\begin{array}{c}\text { Female } \\
\text { Mean }\end{array}$} & \multirow[b]{2}{*}{$\mathrm{SD}$} & \multicolumn{2}{|l|}{ Male } & \multicolumn{2}{|l|}{ Female } & \multicolumn{2}{|l|}{ Male } \\
\hline & & & Mean & SD & Mean & $\mathrm{SD}$ & Mean & $\mathrm{SD}$ \\
\hline$N$ & 2717 & & 2113 & & 570 & & 334 & \\
\hline Age (yrs.) & 72.4 & 5.4 & 73.4 & 5.7 & 70.8 & 5.7 & 70.4 & 5.9 \\
\hline $\mathrm{BMI}(\mathrm{Kg} / \mathrm{M} \mathrm{sq})$ & 26.5 & 4.8 & 26.4 & 3.6 & 29.8 & 5.9 & 26.9 & 4.0 \\
\hline $\mathrm{WC}(\mathrm{cm})$ & 91.1 & 13.4 & 97.8 & 10.1 & 100.5 & 15.6 & 97.6 & 11.3 \\
\hline \% Current Smoker & 12.1 & 32.6 & 9.4 & 29.2 & 13.0 & 33.7 & 19.8 & 39.9 \\
\hline$\%$ Lost $10+\mathrm{lbs}$ & 11.4 & 31.8 & 12.4 & 33.0 & 15.1 & 35.8 & 12.9 & 33.5 \\
\hline $\mathrm{YOL}^{*}$ & 9.1 & 2.0 & 8.3 & 2.6 & 6.5 & 1.3 & 6.2 & 1.7 \\
\hline SPL & 6.4 & 3.2 & 6.4 & 3.2 & 4.8 & 2.2 & 4.5 & 2.3 \\
\hline FLW & 8.3 & 2.6 & 7.7 & 3.0 & 6.0 & 1.8 & 5.6 & 2.0 \\
\hline SOC & 3.5 & 3.2 & 3.0 & 3.0 & 2.3 & 2.4 & 2.2 & 2.3 \\
\hline DEP & 7.0 & 3.2 & 6.9 & 3.2 & 4.5 & 2.4 & 4.6 & 2.4 \\
\hline BED & 8.5 & 2.4 & 7.9 & 2.8 & 5.9 & 1.7 & 5.7 & 1.9 \\
\hline FLUSH & 5.5 & 3.6 & 5.3 & 3.5 & 3.3 & 2.7 & 3.1 & 2.7 \\
\hline COG & 5.8 & 3.8 & 4.9 & 3.8 & 2.2 & 2.7 & 2.1 & 2.5 \\
\hline HOSP & 8.1 & 2.4 & 7.2 & 2.9 & 5.7 & 1.7 & 5.4 & 1.9 \\
\hline EVGG & 6.9 & 3.4 & 6.4 & 3.5 & 3.8 & 2.6 & 4.0 & 2.7 \\
\hline LEV & 5.7 & 3.1 & 5.5 & 3.1 & 3.4 & 2.5 & 3.7 & 2.6 \\
\hline EXSTR & 5.7 & 3.4 & 6.6 & 3.3 & 3.3 & 2.6 & 4.8 & 2.4 \\
\hline IADL & 5.8 & 3.5 & 6.0 & 3.5 & 3.6 & 2.6 & 4.4 & 2.5 \\
\hline BLOCK & 2.4 & 2.8 & 3.4 & 3.1 & 1.0 & 1.7 & 1.7 & 2.1 \\
\hline $\mathrm{ADL}$ & 7.2 & 3.1 & 7.0 & 3.2 & 4.5 & 2.4 & 5.1 & 2.3 \\
\hline TWLK & 4.8 & 3.7 & 5.2 & 3.6 & 2.3 & 2.5 & 3.1 & 2.6 \\
\hline
\end{tabular}

${ }^{*}$ Rows YOL through TWLK present average YHL, calculated from the column variable.

weight was also significantly associated with higher age and smoking $(P<.001)$, but not with weight loss $(P=.679)$.

The lines in Table 3 labeled YOL through TWLK show the (unadjusted) mean YHL for each definition of "healthy," ordered approximately by the increasing difference in YHL between Normal and Overweight BMI. For example, in the ten years after baseline, Normal weight women averaged 7.5 years with no ADL disability. Overweight women averaged 7.4 YHL (from ADL) indicating that they had 0.1 fewer YHL (from ADL) than persons with Normal weight (unadjusted results). As seen in in the appendix Table 5, in the 7 years after baseline, black women with Normal BMI (shown in the lower half of the table) averaged 4.6 years without ADL disability. Table 6 in the appendix shows similar information for persons classified by WC instead of BMI.

3.2. Regression Results. The coefficient for "Overweight" in the regressions is the adjusted difference in YHL between Overweight and Normal, with positive values favoring Overweight. We are interested in the coefficient signs (positive or negative), their sizes, the patterns across variables and sex/race groups, and the statistical significance of the coefficients. It is easier to see the signs, sizes and patterns in a graph. Figure 1 shows the regression coefficients for all YHL measures, by race and sex. To permit easier assessment of the patterns, the variables that turned out to be most favorable to the Overweight are shown at the left and the least favorable at the right. For example, in the topmost panel, which displays results for white women, the coefficient for "YOL" (at the far left) is near zero, meaning that adjusted mortality was similar for the Overweight and the Normal BMI groups. At the far right, the coefficient for TWLK was -.36 , meaning that Overweight persons averaged 0.36 fewer YHL (from TWLK) than persons of Normal weight; that is, 0.36 fewer years in which they were walking 15 feet in 10 seconds or less. The coefficients for white women tended to be small but negative. The trends were quite different for white men, black women, and black men, where the coefficients were also small but were usually positive, indicating that persons with Overweight BMI had somewhat higher YHL than persons with Normal BMI.

Figure 2 shows the difference in YHL between Overweight and Normal weight when adiposity was classified by WC. The trends are similar to those in Figure 1. (Note that the $y$ axis is slightly different in the two figures.) The preponderance of coefficients is again negative for white women, but positive for the other groups.

Columns 1 and 3 of Table 4 contain the regression coefficients shown in Figure 1. The coefficients that are statistically significant are marked with asterisks $\left({ }^{*} P<.10\right.$ 
TABLE 3: Means by BMI category (white women only).

\begin{tabular}{lccc}
\hline & \multicolumn{3}{c}{$\begin{array}{c}\text { BMI Category* } \\
\text { Overwt }\end{array}$} \\
\hline Sample Size & 1037 & 1069 & Obese \\
Age (mean) & 73.0 & 72.4 & 71.3 \\
BMI (mean) & 22.2 & 26.8 & 33.6 \\
WC (cm) & 81.0 & 92.2 & 106.5 \\
\% Current Smoker & 15.7 & 10.0 & 9.5 \\
\% Lost 10+ lbs & 12.6 & 11.3 & 9.7 \\
YOL & 9.1 & 9.1 & 9.1 \\
SPL & 6.3 & 6.6 & 6.3 \\
FLW & 8.2 & 8.4 & 8.3 \\
SOC & 3.4 & 3.5 & 3.7 \\
DEP & 7.0 & 7.0 & 6.8 \\
BED & 8.5 & 8.5 & 8.4 \\
FLUSH & 5.6 & 5.8 & 5.0 \\
COG & 5.6 & 5.8 & 5.9 \\
HOSP & 8.1 & 8.2 & 8.0 \\
EVGG & 7.0 & 7.1 & 6.4 \\
LEV & 5.8 & 5.7 & 5.5 \\
EXSTR & 5.7 & 5.8 & 5.3 \\
IADL & 6.1 & 5.9 & 5.0 \\
BLOCK & 2.8 & 2.4 & 1.7 \\
ADL & 7.5 & 7.4 & 6.6 \\
TWLK & 5.1 & 5.0 & 3.9 \\
\hline
\end{tabular}

${ }^{*}$ Normal $=18.5-24.9$, Overweight $=25-29.9$, Obese $=30+$.

for a 2 -sided test, which is equivalent to $P<.05$ for a 1 sided alternative; $\left.{ }^{* *} P<.05 ;{ }^{* * *} P<.01\right)$. For example, white men who had Overweight BMI averaged 0.26 more years of life (YOL) and 0.32 more years of being satisfied with the purpose of life (SPL) than the Normal weight, both significant at the $P<.05$ level. Most coefficients were not significantly different from zero. Columns 2 and 4, for WC, show the coefficients corresponding to Figure 2. More than half of the coefficients were significantly negative for white women, but coefficients were rarely statistically significant for black women or for men.

Although this paper is primarily about Overweight, Table 7 in the appendix also presents the regression coefficients for Obese BMI and WC. About half of those regression coefficients were significantly different from zero, and all but one were negative, indicating that Obese persons tended to have fewer YHL than persons of Normal weight.

\section{Summary and Discussion}

4.1. Overall. This paper examined the relation between baseline adiposity and future years of healthy life in older adults. Differences in YHL between adiposity categories were examined for 16 measures of YHL, in 4 race by sex groups, using two measures of adiposity (BMI and WC). Regression coefficients, representing the adjusted difference in YHL between Overweight and Normal, were significantly positive for 16 of 128 comparisons, significantly negative for 16, and not significantly different from zero for the remaining 98 coefficients. The "Overweight Paradox," the finding of little difference between Normal and Overweight, thus seemed to hold for various measures of health status as well as for mortality. Obesity was significantly associated with worse outcomes than Normal weight in about half of the comparisons. We next discuss the relevant literature and then consider how the results vary by features of the study design.

4.2. Comparisons with the Literature. As reviewed in [2], many studies have found that the Overweight do not have higher mortality than the Normal weight, consistent with our findings. With respect to outcomes other than mortality, cross-sectional studies in the elderly have found associations between higher BMI and worse morbidity, functional status, and quality of life [14]. Fewer longitudinal studies of older adults are available for outcomes other than mortality. Most of these have focused on activities of daily living (ADL), with mixed results, as was also found here [15-23]. Other important dimensions of health have been studied in less detail. Previous longitudinal analyses have studied the association of adiposity with self-rated health, [22, 24] years without work disability, hospitalization for coronary heart disease, long-term medication, [25] MI, arthritis, diabetes [21], dementia [26], and a new ADL disability [27]. These studies usually found higher risks for obese individuals, but mixed results for the overweight, which is consistent with the results of this paper. None of the studies used years of healthy life, as defined here, and direct comparisons are not possible.

4.3. BMI and WC. The literature has suggested that WC, rather than $\mathrm{BMI}$, should be used to measure adiposity for older adults [2, 6]. BMI may not perform well for several reasons. An increase in body fat can be masked by an age-associated decrease in lean body mass. A person could thus have a stable BMI despite increasing body fat and decreasing muscle mass. Body fat also tends to have a different distribution for older adults, with visceral fat increasing with age. In addition, the usual BMI categories of "underweight," "normal," "overweight," and "obese" were derived using mortality data on younger persons, and the thresholds may not be relevant for older adults.

Here, BMI and WC were fairly similar as measures of adiposity, with two thirds of the persons categorized the same way by either measure, and few large discrepancies. In Table 4, results based on BMI were more favorable to Overweight than results based on WC. (BMI had 14 significantly positive and 5 significantly negative coefficients, compared with 2 positive and 11 negative for WC). This may support recent findings that measures of central obesity are better predictors of survival than BMI [6]. These differences may also be in part because the BMI thresholds were the same for all persons, while the WC thresholds used here were sex and race specific. In addition, traditional BMI thresholds were based on mortality data, while the WC thresholds were defined by tertiles. In an unreported preliminary analysis we 
TABLE 4: Adjusted difference between Overweight and Normal (years) (negative values favor Normal weight).

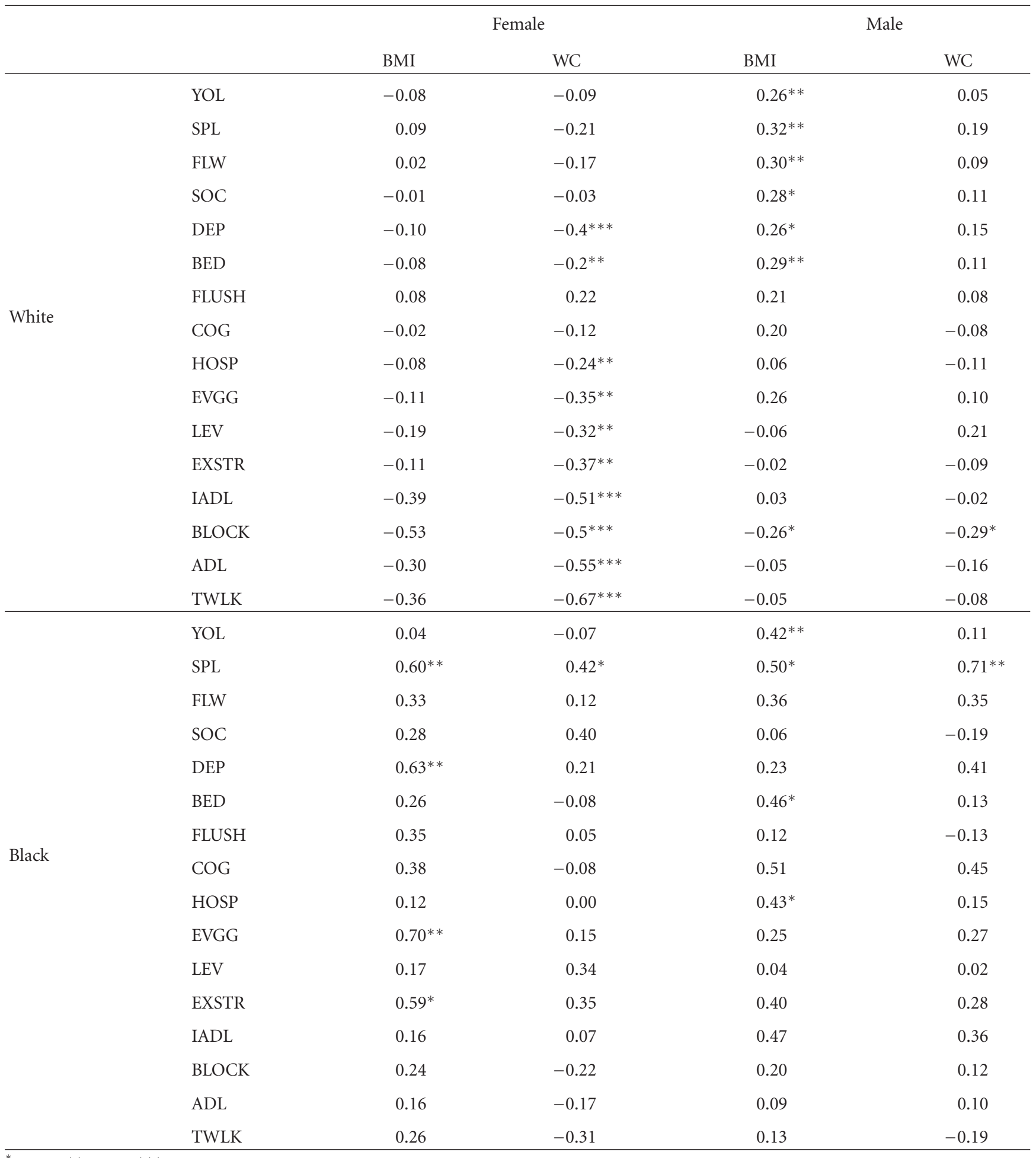

${ }^{*} P<.10 ;{ }^{* *} P<.05 ;{ }^{* * *} P<.01$.

created tertiles of BMI, and found that their association with YHL was similar to that of the traditional categories.

4.4. Different Measures of Health. This analysis used 16 different definitions of YHL, some of which were previously known to be associated with adiposity and others which had not been studied in this way. SPL, FLW, SOC, YOL, and DEP had the least negative (or most positive) associations with Overweight, while EXSTR, IADL, BLOCK, ADL, and TWLK had the most negative associations. If we combine 
the results for Table 4 (Overweight) and Table 7 in the appendix (Obese), SPL had the largest number of significant positive associations ( 5 of 16 coefficients), followed by DEPR and BED (2 each). The highest numbers of significant negative coefficients were for BLOCK (11), ADL and TWLK (9 each), and IADL (8). The outcomes that favored the Overweight or Obese may be thought of as psychological or socially based, while the most negative outcomes represent primarily physical function. Results thus differed somewhat by the aspect of health that was measured.

4.5. Interpretation of Individual Coefficients. The statistical significance of the coefficients in Table 4 should not be overinterpreted because of the issue of multiple comparisons. Of the 128 coefficients, $10 \%$ or about 13 would have been expected to be significant by chance alone. After a conservative Bonferroni correction, only 4 coefficients remained significant; all were for white women and all were negative (BLOCKS (based on BMI and WC) and IADL, ADL, and TWLK (based on WC)).

Of the 16 variables, some of course had larger coefficients than others. Under the theory of order statistics, [28] however, the largest coefficient was not significantly larger than expected under the null hypothesis that all measures of YHL had a similar relation to adiposity (analysis not shown). Thus, unless the reader had a prior hypothesis about a particular variable and subgroup, the coefficients should be only used to describe patterns rather than to identify the variables most sensitive to adiposity.

The positive regression coefficients indicate cases where being Overweight seemed protective. The review paper discusses possible mechanisms for a protective effect, that are not repeated here [2]. The fact that none of the positive coefficients was significantly different from zero after the Bonferroni correction suggests that some of the positive results might be due to chance. It is important not to overinterpret these results without further confirmation.

4.6. Power. The nonsignificant differences do not, of course, imply that results for Overweight and Normal are identical. Rather, they may be due in part to insufficient sample size, especially for the black subpopulation. With the large number of comparisons, it is prohibitive to discuss power in detail, but one example may be instructive. Assume that a difference of 0.5 additional years of healthy life (6 months) in the following 10 years is clinically important. Based on the standard deviations for ADL in Table 2, the power to detect a difference of 6 months was 0.95 for an analysis with 1000 persons per group (similar to numbers for white women) and 0.44 for an analysis with 150 per group (similar to black men). Thus, the study had power to detect meaningful differences between Normal and Overweight, especially for white men and women (calculations not shown). Note that Table 4 has only a handful of coefficients greater than 0.5, suggesting that even with larger samples, any significant differences might not be clinically important.
4.7. Sex and Race. As expected, women had higher YOL (survival) than men. The unadjusted data found that women often (but not always) had higher YHL as well. (see Table 2 and Tables 5 and 6 in the appendix). Overweight was negatively associated with the YHL measures for white women but was usually nonsignificant or positive for white men and for black men and women. As expected from their larger sample size, white women had more statistically significant results than the other groups. But this does not explain why results for white women were more negative. Being Overweight may have more biological consequences for white women than for other groups, perhaps related to differences in the distribution of visceral adipose fat by sex and race [29]. Alternatively, most of the health measures were self-assessed. If, for some reason, white women were more likely than the others to consider being overweight as a negative health characteristic, then Overweight white women might have downrated their health for that reason. Arguing against this response bias explanation, however, is the finding that YHL based on the timed walk, which was not selfreported, was also negatively associated with Overweight. The WC thresholds were lower for white women than for the other groups, but that would not seem to explain why white women had more negative results. It is interesting that even though white women had the smallest WCs and the best outcomes, the results suggest that Overweight white women might benefit the most from losing weight. (Weight loss was not, however, studied in this analysis).

These results should be considered as exploratory rather than definitive, for several reasons. The sex and race differences were not tested formally because the regressions were performed separately by sex and race. (That choice was made because preliminary analyses did find significant interactions between race, sex, adiposity, and outcomes.) In addition, the results are not directly comparable for blacks and whites because of the greater sample size and longer followup for whites. Finally, only three of the four study communities recruited a supplemental cohort of blacks, so the black and white groups are not geographically comparable.

4.8. Implications. The consistent finding that Overweight older adults had similar outcomes to those of Normal weight, based either on mortality or on years of healthy life, suggests strongly that the usual adiposity classifications are inappropriate for older adults, both in the thresholds used and in the labels given to the categories. For older adults, "Normal" BMI is far from normal since the plurality of older adults fall in the "Overweight" category. The pejorative label "Overweight" also seems inappropriate because Overweight and Normal had very similar YHL. Better classifications and labels are needed for older adults. The new standards might be based on BMI, WC, or perhaps on a combination of BMI and WC. (Combined measures of adiposity were not considered here.) The finding that the outcomes differed by sex and race strongly suggests that any clinical guidelines should be specific to age, sex, and race. Also in need of a better label is the so-called "obesity paradox," which might better be referred to as 


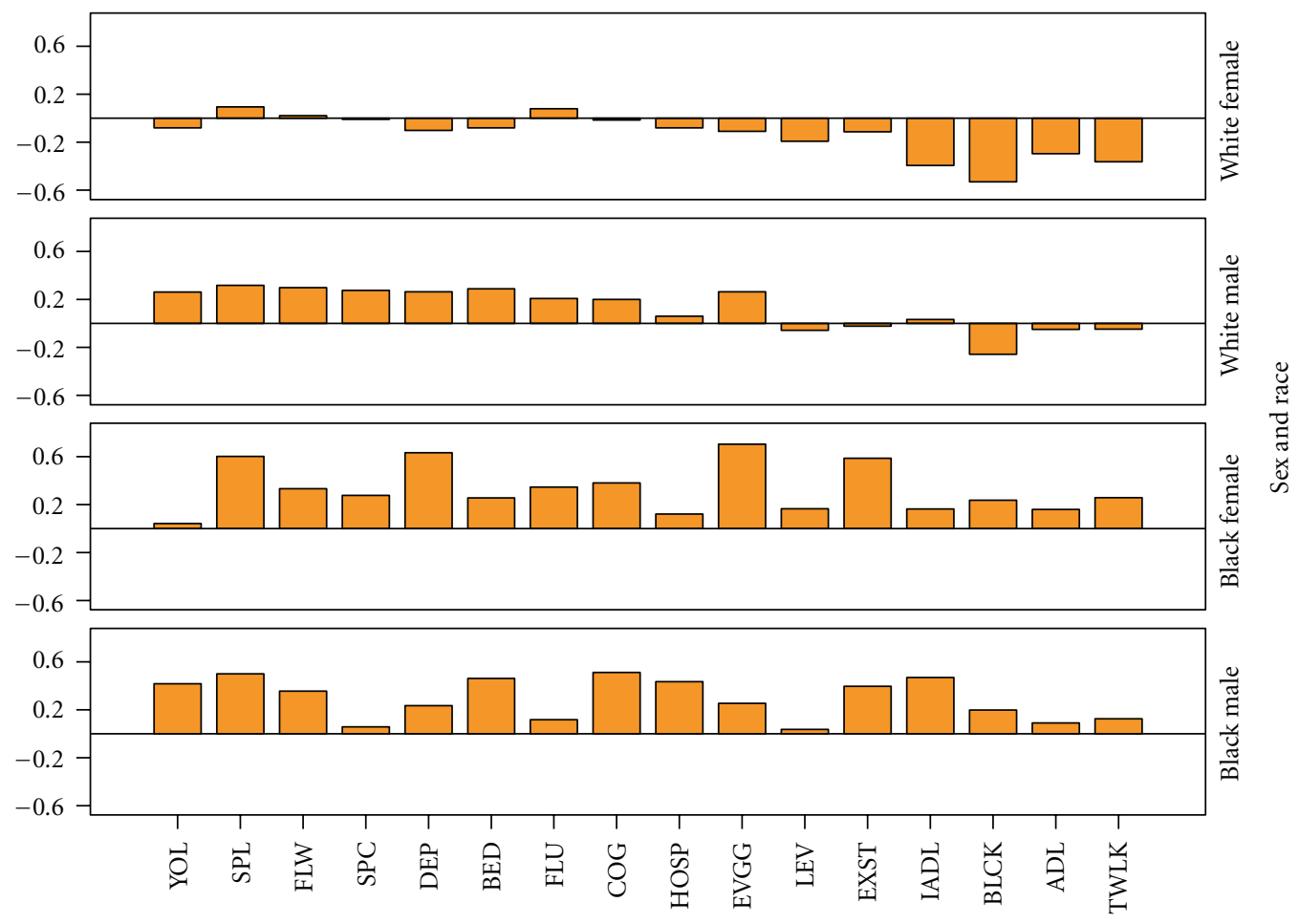

Health measure

FIgURE 1: Adjusted difference in YHL (Overweight BMI - Normal BMI).

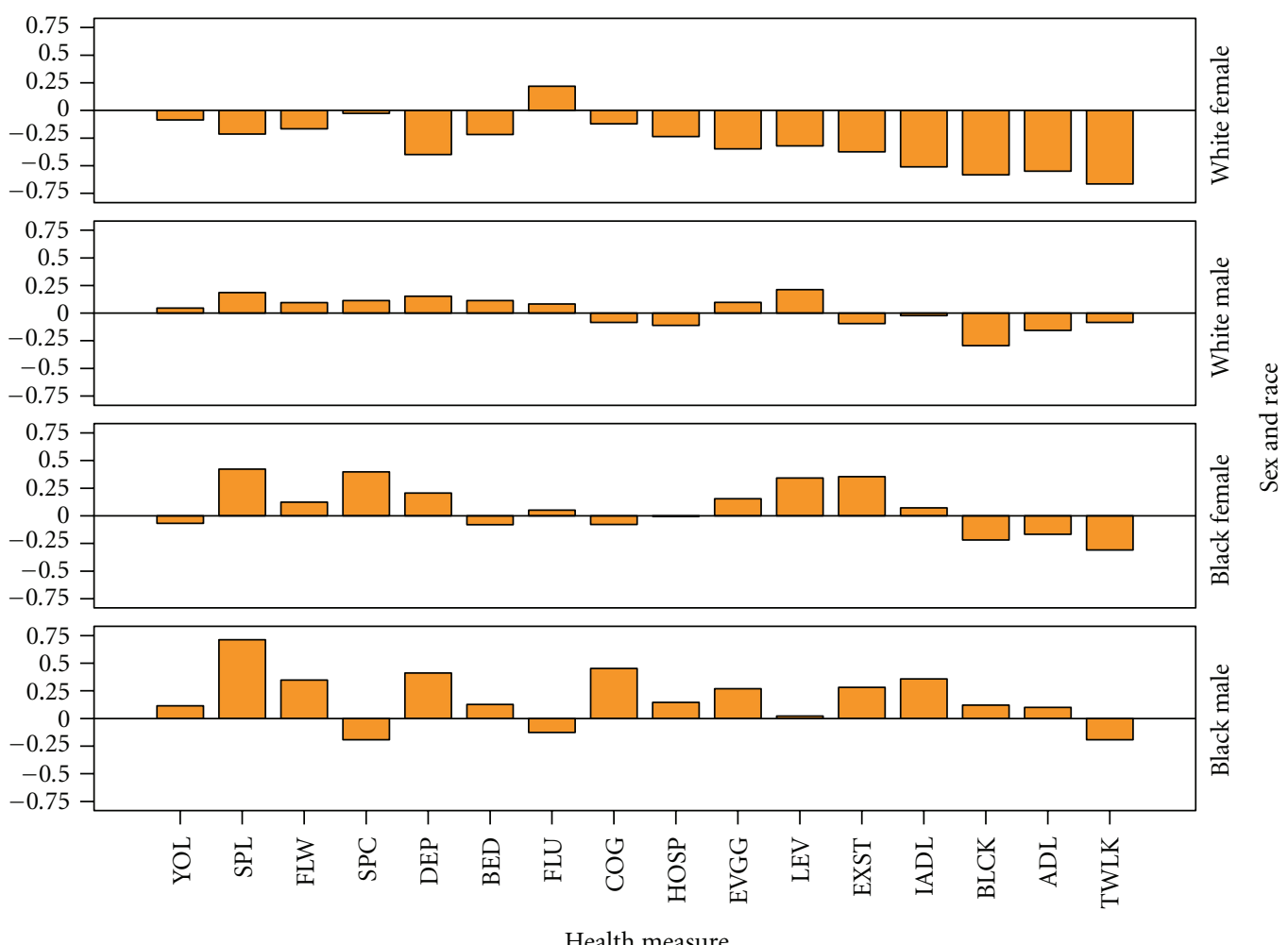

FIgURE 2: Adjusted difference in YHL (Overweight WC - Normal WC). 
the "overweight paradox." Since the Obese had significantly fewer YHL than the Normal weight about half the time, there may be no paradox at all; that is, higher adiposity is deleterious, but the usual thresholds are inappropriate for older adults.

Although we did not specifically study benefits of weight loss, our findings do not support any over-all recommendations for Overweight older adults to lose weight. However, results did vary somewhat by sex and race, and also by the definition of years of healthy life. Increasingly, clinicians have recognized the importance of engaging patients in defining what outcomes matter to them, using a "personcentered medicine" approach [30]. Our results encourage clinicians to consider not only objective health measures like mortality, cholesterol, and blood pressure in making decisions about weight loss, but also to reflect on health and quality of life as defined by the patient. The domains of health that matter to the patient, some of which we examined here, can become the basis for anticipating benefits and agreeing on any plans for weight loss. Rather than assuming that weight loss confers general benefits to all overweight or obese individuals, providers can engage patients in defining ways of maximizing each patient's own benefits.

4.9. Study Strengths. The main strength of this study is its high quality longitudinal data (10 years) on 16 different health outcomes for older adults, as a function of measured BMI and WC. Tables 5 and 6 in the appendix should be useful to other investigators in this area, since they present information not generally available.

4.10. Limitations. The regression analyses used here might not be considered ideal for some of the outcome variables. We chose to perform the same analysis for all of the variables, to allow comparisons. Issues of causality (e.g., whether adiposity affects physical activity or physical activity affects adiposity) were not addressed. The large number of regression coefficients presented makes it unwise to emphasize any particular coefficient, but consistency across the race and sex groups may support future confirmatory research. Standard errors for the regression coefficients are available from the authors. Only 3 communities had an enriched sample of blacks, limiting comparison of blacks to whites. The analysis does not identify the optimal BMI or WC, and classifications that used BMI and WC jointly were not considered. Different choices for the thresholds used to dichotomize the outcome variables would have changed mean YHL but probably have little effect on the difference between Normal and Overweight. We did not create a composite summary of all 16 variables because our purpose was to emphasize the different dimensions of health.

4.11. Conclusions. Overweight older Americans lived as long as Normal weight persons and usually experienced as many years of healthy life, as defined by 16 measures of health. Thus, the Overweight Paradox was seen to hold generally, especially for men and black women, and for domains of health other than physical function. Weight loss recommendations for older adults should be tailored to the appropriate sex and race group and may not be necessary for the Overweight. If one accepts that only Obese older adults are at risk for negative health consequences from their weight, then only about a fourth of older adults may require attention or treatment. The so-called obesity epidemic for older adults may be less severe than is usually supposed. Further research should develop optimal levels of BMI and WC for older adults, which may differ substantially by sex and race, and by the criterion measure of health status used.

\section{Appendix}

\section{Missing Data}

In CHS, most measures were taken annually from 1990 to 1999. However, self-rated health was measured semiannually from 1990 to 2005, and mortality was known (for the current analysis) through 2007. We imputed missing selfrated health data and used that, where necessary, to help impute data for the other variables. For self-rated health, we coded the original response categories (excellent, very good, good, fair, poor) as 95, 90, 80, 30, and 15 [31]. These values represent the approximate percent probability that a person in that state would be in excellent, very good, or good health in the following year. Under the assumption that a dead person is not healthy and will not be healthy next year either, we assigned a value of 0 to observation that were not made because the person had died. After this recoding, we imputed missing data by linear interpolation over time, whenever there was a valid value before and a valid value or death after the missing data. The remaining unimputed data, for persons alive but missing at the end of the sequence, used the last observation carried forward. Because so much information was collected after 1999, we rarely had to impute missing data from 1990 to 1999 by extrapolation (less than $0.3 \%$ of the time). We are thus comfortable with the imputation for self-rated health data during the study period.

To impute missing data for the other variables, each variable was transformed to a new scale representing the probability of being in excellent, very good, or good health; deaths were set to zero; and data were interpolated. Data missing at the end of the sequence for persons still alive was imputed as the average of the last available observation and the estimate from self-rated health at that time (both on the same scale because of the transformation). After imputation, the variables were transformed back to the original scales.

Missing data for three variables were imputed differently. Receiving a flu shot was not associated with self-rated health. Social support and life events were not measured as often as the other variables. For these variables, we used a regression of the person's known values on the logarithm of time from the last measure to impute missing data, as illustrated elsewhere [32]. 
Table 5: Means by BMI*, gender, and race.

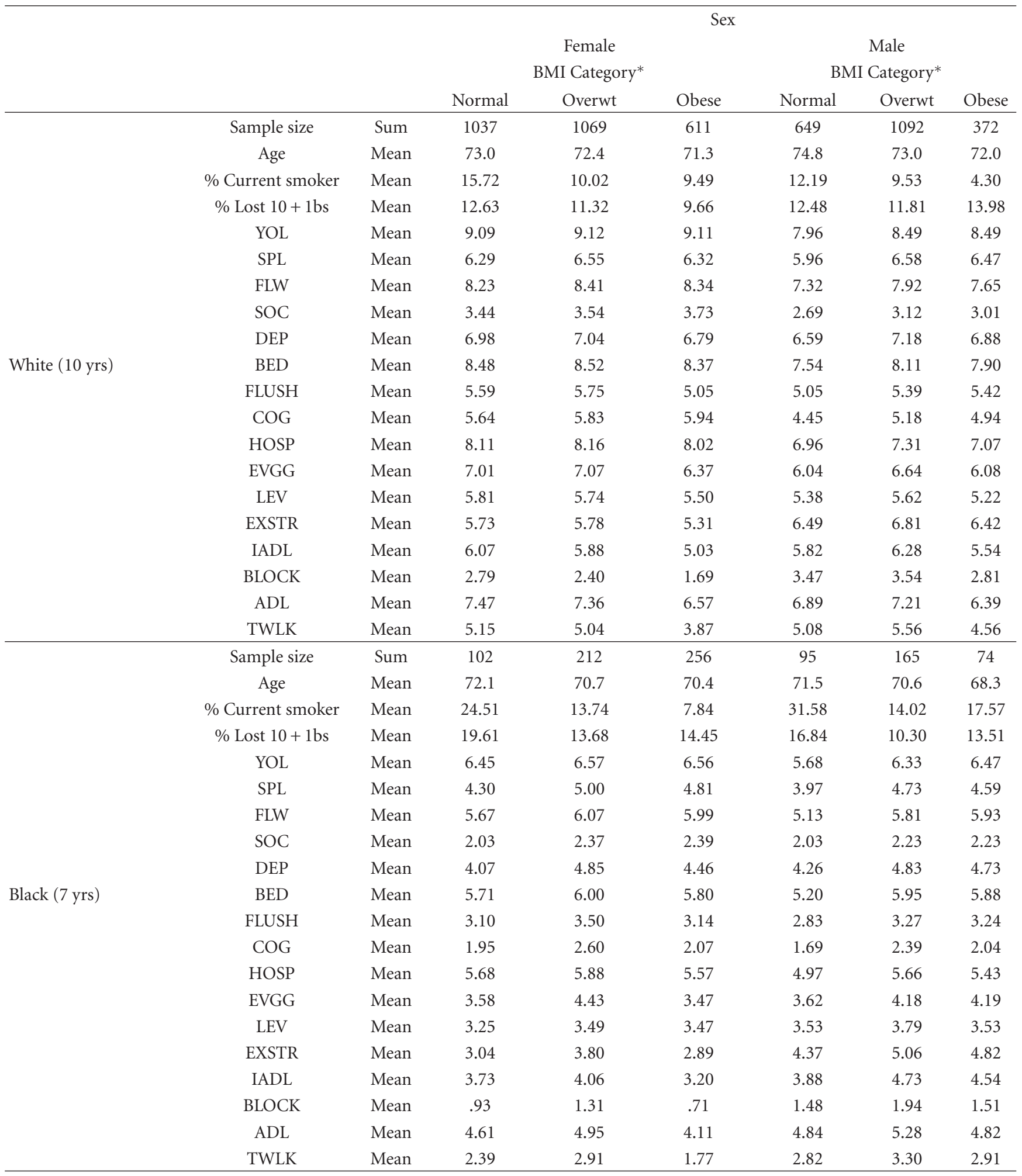

${ }^{*}$ Normal $=18.5-24.9($ normal $)$, Overweight $=25-29.9($ overweight $)$, Obese $=30+$. 
Table 6: Means by Waist Circumference*, Gender and Race.

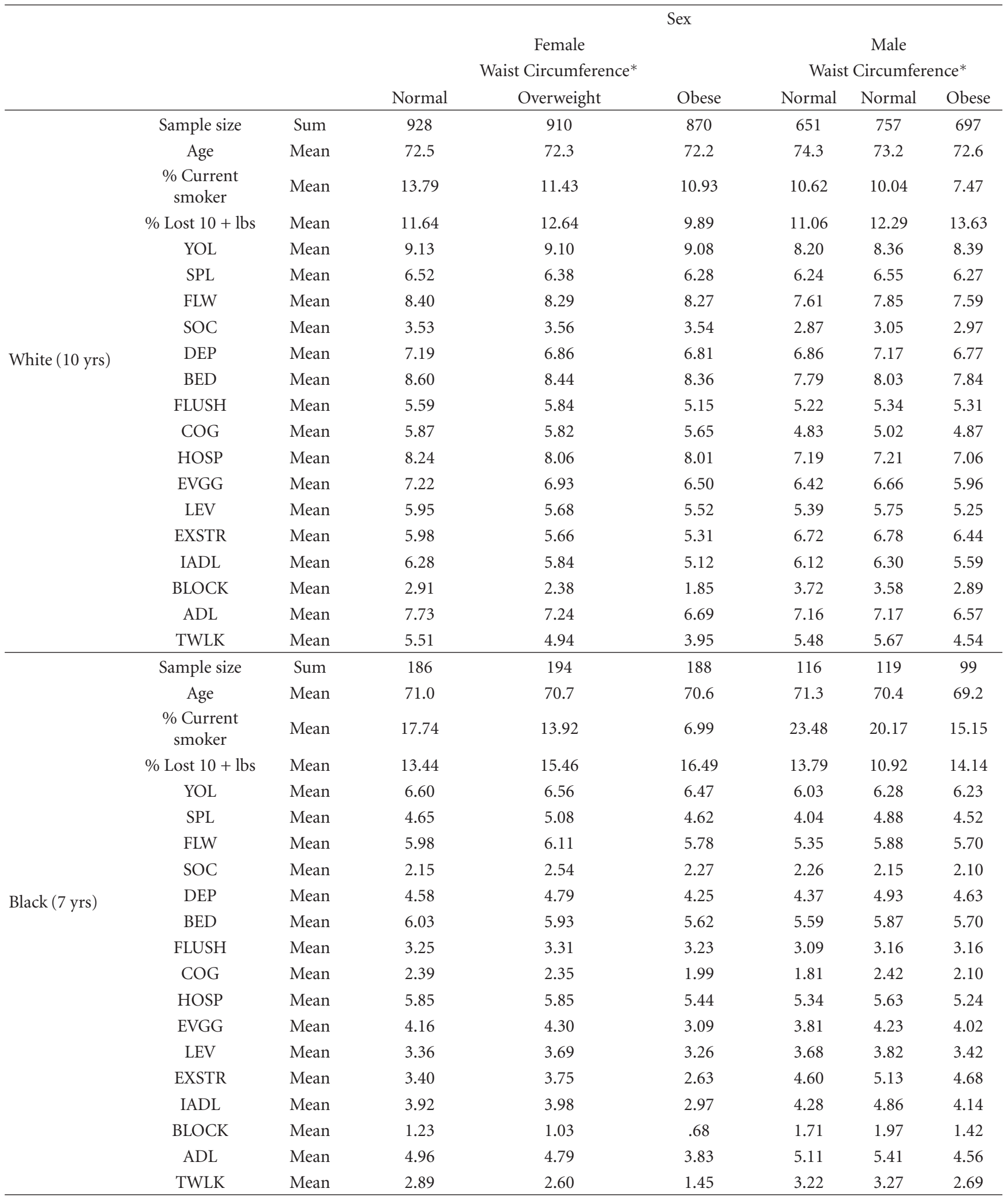

* Overweight $=84.5-96.5$ for White Female, 94.0-107.5 for Black Female, 93.0-101.5 for Male. 
TABLE 7: Adjusted difference between Obese and Normal YHL (in years; negative values favor Normal).

\begin{tabular}{|c|c|c|c|c|c|}
\hline & \multicolumn{2}{|c|}{ Female } & \multicolumn{2}{|c|}{ Male } \\
\hline & & BMI & WC & BMI & WC \\
\hline \multirow{16}{*}{ White } & YOL & $-0.21^{* *}$ & -0.12 & 0.10 & -0.02 \\
\hline & SPL & $-0.32^{* *}$ & -0.34 & 0.02 & -0.20 \\
\hline & FLW & $-0.22^{*}$ & $-0.21^{*}$ & -0.17 & $-0.27^{*}$ \\
\hline & SOC & 0.07 & -0.05 & 0.05 & -0.03 \\
\hline & DEP & $-0.53^{* * *}$ & $-0.48^{* * *}$ & -0.25 & $-0.35^{* *}$ \\
\hline & BED & $-0.38^{* * *}$ & $-0.32 * * *$ & -0.10 & -0.17 \\
\hline & FLUSH & $-0.70 * * *$ & $-0.48^{* * *}$ & 0.13 & -0.01 \\
\hline & $\mathrm{COG}$ & -0.26 & $-0.33^{* *}$ & $-0.42^{*}$ & $-0.42^{* *}$ \\
\hline & HOSP & $-0.39 * * *$ & $-0.32 * * *$ & $-0.36^{* *}$ & $-0.36^{* *}$ \\
\hline & EVGG & $-1.02^{* * *}$ & $-0.83^{* * *}$ & $-0.51^{* *}$ & $-0.71^{* * *}$ \\
\hline & LEV & $-0.58^{* * *}$ & $-0.51^{* * *}$ & $-0.63^{* * *}$ & $-0.38^{* *}$ \\
\hline & EXSTR & $-0.80^{* * *}$ & $-0.77 * * *$ & $-0.63^{* * *}$ & $-0.55^{* * *}$ \\
\hline & IADL & $-1.52^{* * *}$ & $-1.28^{* * *}$ & $-0.98^{* * *}$ & $-0.88^{* * *}$ \\
\hline & BLOCK & $-1.44^{* * *}$ & $-1.14^{* * *}$ & $-1.21^{* * *}$ & $-1.10^{* * *}$ \\
\hline & $\mathrm{ADL}$ & $-1.34^{* * *}$ & $-1.15^{* * *}$ & $-1.10^{* * *}$ & $-0.88^{* * *}$ \\
\hline & TWLK & $-1.93^{* * *}$ & $-1.71^{* * *}$ & $-1.39^{* * *}$ & $-1.39^{* * *}$ \\
\hline \multirow{16}{*}{ Black } & YOL & 0.05 & -0.15 & $0.47^{*}$ & 0.03 \\
\hline & SPL & 0.41 & -0.05 & 0.27 & 0.29 \\
\hline & FLW & 0.27 & -0.21 & 0.33 & 0.09 \\
\hline & SOC & 0.28 & 0.12 & 0.02 & -0.27 \\
\hline & DEP & 0.24 & -0.34 & 0.02 & 0.05 \\
\hline & BED & 0.08 & $-0.37^{* *}$ & 0.28 & -0.10 \\
\hline & FLUSH & -0.02 & -0.06 & -0.03 & -0.19 \\
\hline & COG & -0.09 & -0.40 & -0.18 & 0.00 \\
\hline & HOSP & -0.18 & $-0.42^{* *}$ & 0.11 & -0.28 \\
\hline & EVGG & -0.26 & $-1.03^{* * *}$ & 0.13 & 0.00 \\
\hline & LEV & 0.17 & -0.11 & -0.37 & -0.43 \\
\hline & EXSTR & -0.31 & $-0.78^{* * *}$ & -0.03 & -0.22 \\
\hline & IADL & $-0.70^{* *}$ & $-0.89 * * *$ & 0.08 & -0.46 \\
\hline & BLOCK & $-0.37^{*}$ & $-0.57^{* * *}$ & -0.38 & $-0.52^{*}$ \\
\hline & $\mathrm{ADL}$ & $-0.69^{* *}$ & $-1.10^{* * *}$ & -0.53 & $-0.82^{* * *}$ \\
\hline & TWLK & $-0.89^{* * *}$ & $-1.43^{* * *}$ & -0.59 & $-0.89^{* * *}$ \\
\hline
\end{tabular}

${ }^{*} P<.10 ; * * P<.05 ; * * * P<.01$.

\section{Acknowledgment}

The research reported in this article was supported by contracts N01-HC-85239, N01-HC-85079 through N01HC-85086, N01-HC-35129, N01 HC-15103, N01 HC-55222, N01-HC-75150, N01-HC-45133, and Grant HL080295 from the National Heart, Lung, and Blood Institute (NHLBI), with additional contribution from the National Institute of Neurological Disorders and Stroke (NINDS). Additional support was provided through AG-023629, AG-15928, AG-20098, and AG-027058 from the National Institute on Aging (NIA). A full list of principal CHS investigators and institutions can be found at http://www.chs-nhlbi.org/pi.htm. Dr. Thielke was supported by an NIMH Beeson Career Development Award (K23 MH093591-01).

\section{References}

[1] National Heart, Lund, and Blood Institute, National Institutes of Health, "Clinical Guidelines on the identification, evaluation, and treatment of overweight and obesity in adults," 1998.

[2] A. Oreopoulos, K. Kalantar-Zadeh, A. M. Sharma, and G. C. Fonarow, "The Obesity Paradox in the elderly: potential mechanisms and clinical implications," Clinics in Geriatric Medicine, vol. 25, no. 4, pp. 643-659, 2009.

[3] L. P. Fried, N. O. Borhani, P. Enright et al., "The cardiovascular Health Study: design and rationale," Annals of Epidemiology, vol. 1, no. 3, pp. 263-276, 1991.

[4] D. G. Ives, A. L. Fitzpatrick, D. E. Bild et al., "Surveillance and ascertainment of cardiovascular events: the Cardiovascular Health Study," Annals of Epidemiology, vol. 5, no. 4, pp. 278$285,1995$. 
[5] A. Heiat, V. Vaccarino, and H. M. Krumholz, "An evidencebased assessment of federal guidelines for overweight and obesity as they apply to elderly persons," Archives of Internal Medicine, vol. 161, no. 9, pp. 1194-1203, 2001.

[6] T. A. Welborn and S. S. Dhaliwal, "Preferred clinical measures of central obesity for predicting mortality," European Journal of Clinical Nutrition, vol. 61, no. 12, pp. 1373-1379, 2007.

[7] B. L. Neugarten, R. J. Havighurst, and S. S. Tobin, "The measurement of life satisfaction," Journal of Gerontology, vol. 16, pp. 134-143, 1961.

[8] L. M. Martire, R. Schulz, M. B. Mittelmark, and J. T. Newsom, "Stability and change in older adults' social contact and social support: the Cardiovascular Health Study," Journals of Gerontology B, vol. 54, no. 5, pp. S302-S311, 1999.

[9] E. M. Andresen, J. A. Malmgren, W. B. Carter, and D. L. Patrick, "Screening for depression in well older adults: evaluation of a short form of the CES-D (center for Epidemiologic Studies Depression Scale)," American Journal of Preventive Medicine, vol. 10, pp. 77-84, 1994.

[10] E. L. Teng and H. C. Chui, "The Modified Mini-Mental State (MMS) examination," Journal of Clinical Psychiatry, vol. 48, no. 8, pp. 314-318, 1987.

[11] R. H. Rahe and R. J. Arthur, "Life change and illness studies: past history and future directions," Journal of Human Stress, vol. 4, no. 1, pp. 3-15, 1978.

[12] P. Diehr and C. Hirsch, "Health benefits of increased walking for sedentary, generally healthy older adults: using longitudinal data to approximate an intervention trial," Journals of Gerontology A, vol. 65, no. 9, pp. 982-989, 2010.

[13] T. Lumley, P. Diehr, S. Emerson, and L. Chen, "The importance of the normality assumption in large public health data sets," Annual Review of Public Health, vol. 23, pp. 151-169, 2002.

[14] A. Zajacova, J. B. Dowd, and S. A. Burgard, "Overweight adults may have the lowest mortality-do they have the best health?" American Journal of Epidemiology, vol. 173, no. 4, pp. 430-437, 2011.

[15] S. Walter, A. Kunst, J. MacKenbach, A. Hofman, and H. Tiemeier, "Mortality and disability: the effect of overweight and obesity," International Journal of Obesity, vol. 33, no. 12, pp. 1410-1418, 2009.

[16] M. Reuser, L. G. Bonneux, and F. J. Willekens, "Smoking kills, obesity disables: a multistate approach of the US health and retirement survey," Obesity, vol. 17, no. 4, pp. 783-789, 2009.

[17] I. A. Lang, D. J. Llewellyn, K. Alexander, and D. Melzer, "Obesity, physical function, and mortality in older adults," Journal of the American Geriatrics Society, vol. 56, no. 8, pp. 1474-1478, 2008.

[18] S. L. Reynolds and J. M. McIlvane, "The impact of obesity and arthritis on active life expectancy in older Americans," Obesity, vol. 17, no. 2, pp. 363-369, 2009.

[19] E. W. Gregg and J. M. Guralnik, "Is disability obesity's price of longevity?" Journal of the American Medical Association, vol. 298, no. 17, pp. 2066-2067, 2007.

[20] S. Al Snih, K. J. Ottenbacher, K. S. Markides, Y. F. Kuo, K. Eschbach, and J. S. Goodwin, "The effect of obesity on disability vs mortality in older Americans," Archives of Internal Medicine, vol. 167, no. 8, pp. 774-780, 2007.

[21] I. Janssen, "Morbidity and mortality risk associated with an overweight BMI in older men and women," Obesity, vol. 15, no. 7, pp. 1827-1840, 2007.

[22] P. Diehr, E. S. O’Meara, A. Fitzpatrick, A. B. Newman, L. Kuller, and G. Burke, "Weight, mortality, years of healthy life, and active life expectancy in older adults," Journal of the American Geriatrics Society, vol. 56, no. 1, pp. 76-83, 2008.

[23] S. L. Reynolds, Y. Saito, and E. M. Crimmins, "The impact of obesity on active life expectancy in older American men and women," Gerontologist, vol. 45, no. 4, pp. 438-444, 2005.

[24] P. Diehr, A. B. Newman, S. A. Jackson, L. Kuller, and N. Powe, "Weight-modification trials in older adults: what should the outcome measure be?" Current Controlled Trials in Cardiovascular Medicine, vol. 3, article no. 1, 2002.

[25] T. L. S. Visscher, A. Rissamen, J. C. Seidell et al., "Obesity and unhealthy life-years in adult Finns," Archives of Internal Medicine, vol. 164, pp. 1413-1420, 2004.

[26] A. L. Fitzpatrick, L. H. Kuller, O. L. Lopez et al., "Midlife and late-life obesity and the risk of dementia: cardiovascular health study," Archives of Neurology, vol. 66, no. 3, pp. 336-342, 2009.

[27] A. M. Arnold, A. B. Newman, M. Cushman, J. Ding, and S. Kritchevsky, "Body weight dynamics and their association with physical function and mortality in older adults: the cardiovascular health study," Journals of Gerontology A, vol. 65, no. 1, pp. 63-70, 2010.

[28] W. J. Dixon and F. J. Massey, Introduction to Statistical Analysis, McGraw Hill, New York, NY, USA, 1957.

[29] S. M. Camhi, G. A. Bray, C. Bouchard et al., "The relationship of waist circumference and BMI to visceral, subcutaneous, and total body fat: Sex and race differences," Obesity, vol. 19, no. 2, pp. 402-408, 2011.

[30] J. E. Mezzich, J. Snaedal, C. Van Weel, M. Botbol, and I. Salloum, "Introduction to person-centred medicine: from concepts to practice," Journal of Evaluation in Clinical Practice, vol. 17, no. 2, pp. 330-332, 2011.

[31] P. Diehr, D. L. Patrick, J. Spertus, C. I. Kiefe, M. McDonell, and S. D. Fihn, "Transforming self-rated health and the SF-36 scales to include death and improve interpretability," Medical Care, vol. 39, no. 7, pp. 670-679, 2001.

[32] P. Diehr, W. E. Lafferty, D. L. Patrick, L. Downey, S. M. Devlin, and L. J. Standish, "Quality of life at the end of life," Health and Quality of Life Outcomes, vol. 5, article no. 51, 2007. 


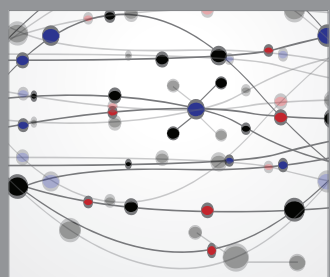

The Scientific World Journal
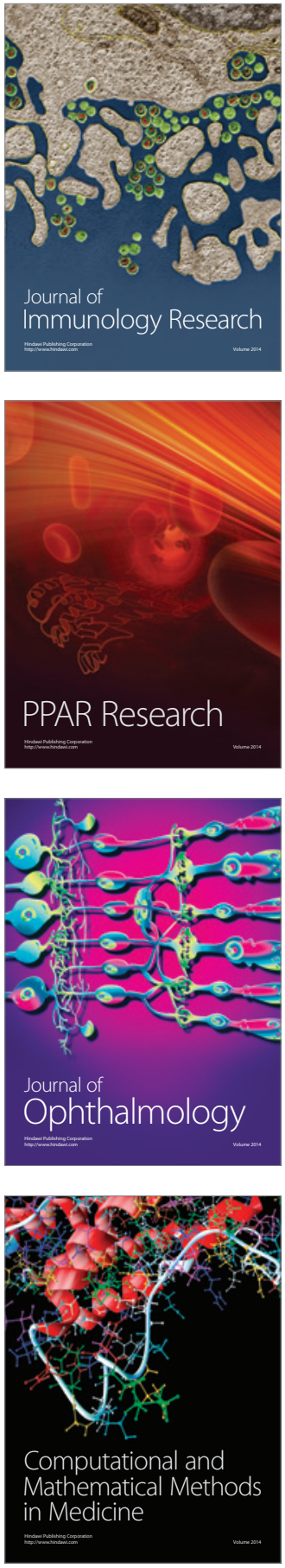

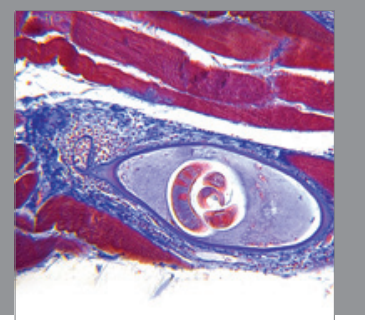

Gastroenterology

Research and Practice
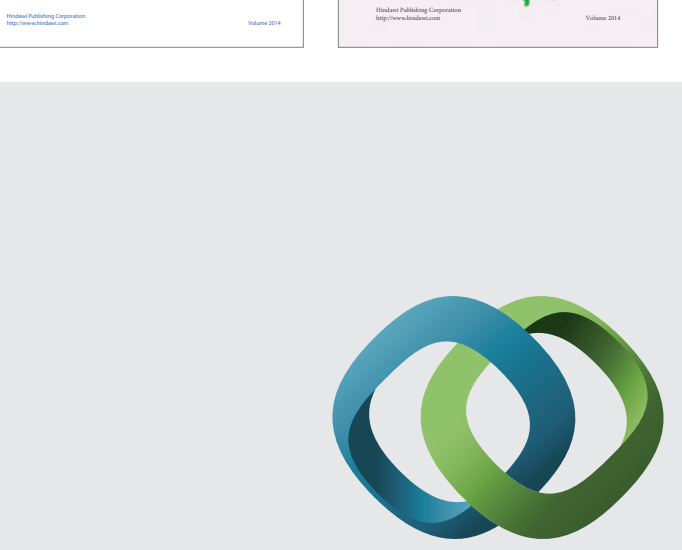

\section{Hindawi}

Submit your manuscripts at

http://www.hindawi.com
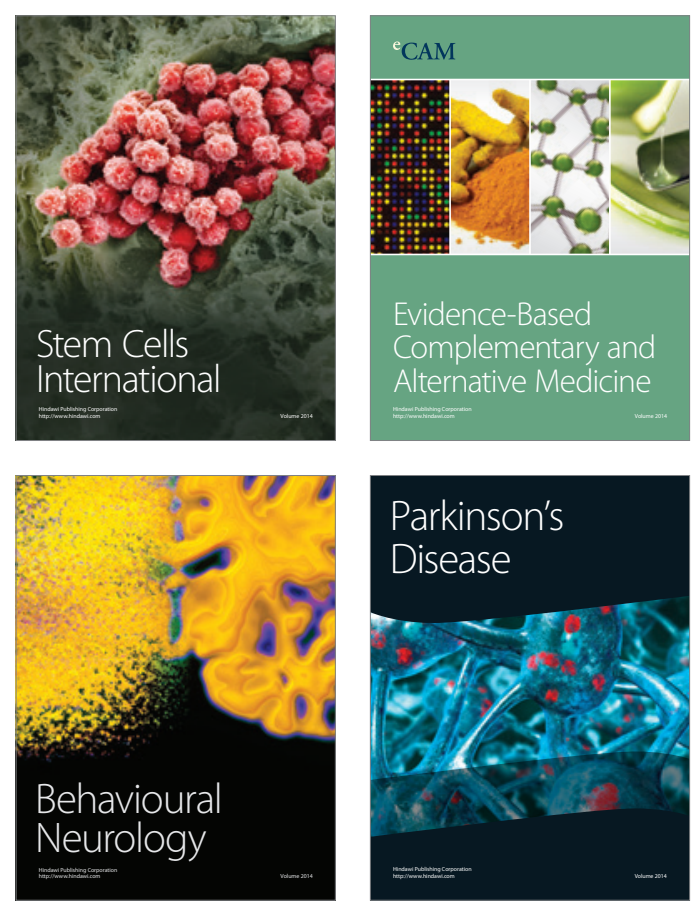

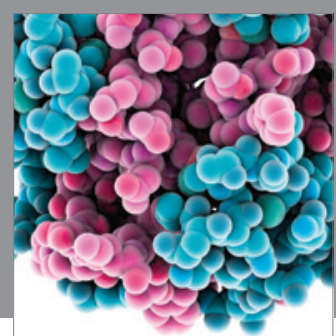

Journal of
Diabetes Research

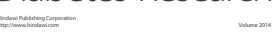

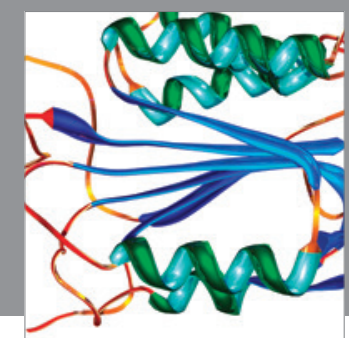

Disease Markers
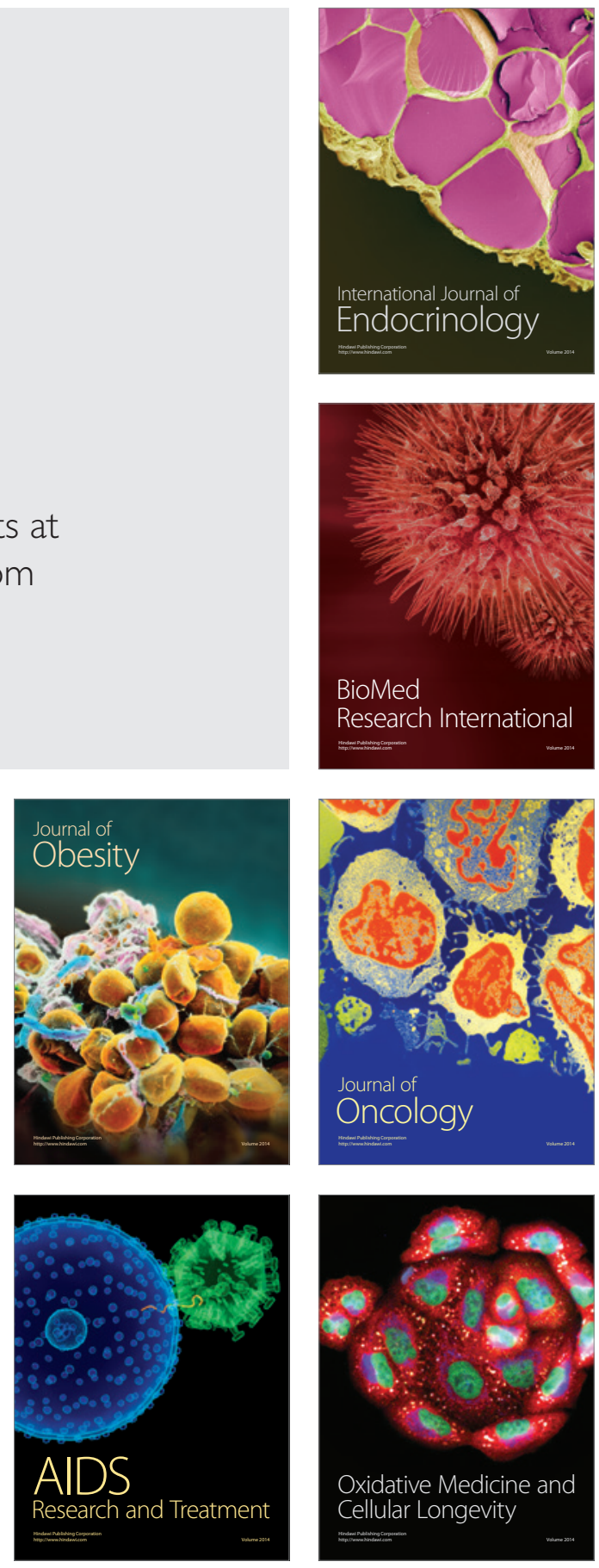